University of Wollongong

Research Online

Faculty of Engineering and Information

Faculty of Engineering and Information

Sciences - Papers: Part A

Sciences

$1-1-2014$

\title{
Analysing the Quality of Experience of multisensory media from measurements of physiological responses
}

Jacob Donley

University of Wollongong, jrd089@uowmail.edu.au

Christian H. Ritz

University of Wollongong, critz@uow.edu.au

Muawiyath Shujau

University of Wollongong, mshujau@uow.edu.au

Follow this and additional works at: https://ro.uow.edu.au/eispapers

Part of the Engineering Commons, and the Science and Technology Studies Commons

Research Online is the open access institutional repository for the University of Wollongong. For further information contact the UOW Library: research-pubs@uow.edu.au 


\title{
Analysing the Quality of Experience of multisensory media from measurements of physiological responses
}

\author{
Abstract \\ This paper investigates the Quality of Experience (QoE) of multisensory media by analysing biosignals \\ collected by electroencephalography (EEG) and eye gaze sensors and comparing with subjective ratings. \\ Also investigated is the impact on QoE of various levels of synchronicity between the sensory effect and \\ target video scene. Results confirm findings from previous research that show sensory effects added to \\ videos increases the QoE rating. While there was no statistical difference observed for the QoE ratings for \\ different levels of sensory effect synchronicity, an analysis of raw EEG data showed $25 \%$ more activity in \\ the temporal lobe during asynchronous effects and $20-25 \%$ more activity in the occipital lobe during \\ synchronous effects. The eye gaze data showed more deviation for a video with synchronous effects and \\ the EEG showed correlating occipital lobe activity for this instance. These differences in physiological \\ responses indicate sensory effect synchronicity may affect QoE despite subjective ratings appearing \\ similar.

\section{Disciplines} \\ Engineering | Science and Technology Studies

\section{Publication Details} \\ J. Donley, C. Ritz \& M. Shujau, "Analysing the Quality of Experience of multisensory media from \\ measurements of physiological responses," in Sixth International Workshop on Quality of Multimedia \\ Experience (QoMEX), 2014, pp. 286-291.
}




\title{
ANALYSING THE QUALITY OF EXPERIENCE OF MULTISENSORY MEDIA FROM MEASUREMENTS OF PHYSIOLOGICAL RESPONSES
}

\author{
Jacob Donley, Christian Ritz, Muawiyath Shujau \\ School of Electrical Computer and Telecommunications Engineering, University of Wollongong, \\ Wollongong, NSW, Australia,2522,jrd089@uowmail.edu.au, critz@uow.edu.au,ms970@uowmail.edu.au
}

\begin{abstract}
This paper investigates the Quality of Experience (QoE) of multisensory media by analysing biosignals collected by electroencephalography (EEG) and eye gaze sensors and comparing with subjective ratings. Also investigated is the impact on QoE of various levels of synchronicity between the sensory effect and target video scene. Results confirm findings from previous research that show sensory effects added to videos increases the QoE rating. While there was no statistical difference observed for the QoE ratings for different levels of sensory effect synchronicity, an analysis of raw EEG data showed 25\% more activity in the temporal lobe during asynchronous effects and 20$25 \%$ more activity in the occipital lobe during synchronous effects. The eye gaze data showed more deviation for a video with synchronous effects and the EEG showed correlating occipital lobe activity for this instance. These differences in physiological responses indicate sensory effect synchronicity may affect QoE despite subjective ratings appearing similar.
\end{abstract}

Index Terms - quality of experience (QoE), multisensory media, gaze tracking (GT), electroencephalography (EEG), synchronicity

\section{INTRODUCTION}

Multisensory media systems provide for an enhanced user Quality of Experience (QoE), which in this context is also known as Sensory Experience [1], through stimulating human senses by vibration, blowing air and ambient lighting effects at time-points that are linked to an audio/visual scene in a multimedia presentation $[2,3]$. Previous research investigating the use of a multisensory media system to provide these sensory effects for video demonstrated an enhanced user experience compared to the same videos without the sensory effects [3]. This was based on subjective testing, where a participant selected their vote from an international standard scale for subjective quality assessment of multimedia [2-4]. In [2, 5], research further investigated the enhancement of specific emotional responses provided by sensory effects added to video sequences. This existing research is based on QoE or emotional response ratings provided by each user at the end of the test stimulus. In contrast, this paper investigates the temporal variation of QoE during the presentation of the sensoryenhanced test stimulus. This is achieved by collecting and analysing physiological responses using biosensors whilst a subject participates in a QoE evaluation of the test sequences. Presented here is a system that integrates an electroencephalography (EEG) headset and an eye gaze tracker within a multisensory media presentation and QoE evaluation system [2, 3].

EEG is a method of reading the electrical activity of the brain by measuring the potential difference between two different receptors positioned on the surface of the scalp. Analysing EEG recordings can be used to identify a person's emotional state for both mapping to the six primary emotions elicited by viewing images [6] as well as distinguishing between like and dislike when viewing video advertisements [7]. Recent research [8] has investigated the use of EEG data for understanding the timevarying QoE of multimedia and research in [9] investigates EEG responses for comparison with user 'tags' added temporally during presentation of the multisensory media at time-points chosen by the user. Eye gaze tracking data collected while users watch a media presentation can be analysed to detect when a user glances or stares at objects of interest within an audio/visual scene. This is directly related to what a human brain is processing when engaged in an activity [10]. Jointly analysing gaze and EEG activity can be used to investigate how a person feels about what they are observing onscreen at that particular time. Such analysis is explored in this work to see how it relates to QoE for multisensory media applications.

Previous research [4] has concluded that different sensory effects have varying impact on the overall QoE and emotion as judged by a participant. Results have also shown that the level of QoE enhancement is related to the genre of the video. Such research provides useful information in regards to modelling sensory effects to ensure QoE is maximised. Poor synchronisation of audio and visual content is known to provide a poor user experience [11, 12]. Recent work [1] has found that synchronicity of olfactory sensory effects with the target video scene may also influence QoE. This paper provides a new investigation into the impact of synchronicity between a video scene and other sensory effects (wind, vibration, and lighting) on the resulting QoE. This is based on analysing subjective QoE ratings as well as biosensor signals (EEG and eye gaze) collected for sequences containing no effects, effects synchronised to the desired time-location and effects asynchronous with the desired time-location.

Section 2 of this paper reviews techniques for EEG and eye gaze monitoring and describes the methods adopted in this research. Section 3 describes the biosensor-based QoE evaluation system while Section 4 describes the multisensory media QoE evaluation incorporating biosensors. Results from subjective QoE testing and analysis of EEG and eye gaze data for a selection of sensory enhanced videos with different types and synchronisation of effects are presented in Section 5 with conclusions provided in Section 6.

\section{MEASURING EMOTIONAL RESPONSES FROM EEG AND EYE GAZE SIGNALS}

This section reviews existing approaches to measuring emotional response from EEG and eye gaze signals and describes the techniques adopted in this work. 


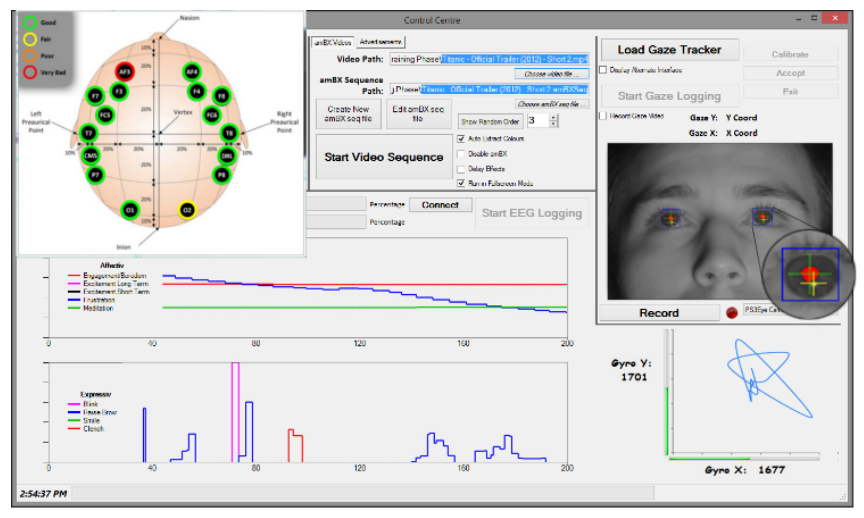

Figure 1: Layout of evaluation system

\subsection{Electroencephalography (EEG)}

Being able to accurately and logically test for emotions and user experiences by collecting EEG data is a problem when most test equipment for these applications can be quite uncomfortable and cumbersome. For example, the standard 10-10 EEG system requires 81 separate wires and corresponding electrodes [13] usually held on a net or cap which is strapped over the head. More recently, less obtrusive EEG headsets have become available such as the Emotiv EEG [14] that is used in this work. This new style of EEG head set fits neatly and comfortably on a person's head and includes a built-in accelerometer to record head movement.

Using multiple sensors (14 channels in the case of the EEG headset used here) allows for recording signals from different parts of the brain. For example the frontal lobe of the cerebral cortex is associated with behavioural and emotional responses, the lobe also functions in close relationship with other regions of the brain in aspects of memory and learning, attention and motivation [15]. All of these complex relationships make it possible to read emotional behaviour from electrodes. As well as recording raw signals, the Emotiv EEG system includes software for deriving parameters from these signals, such as 'frustration' values, that can be linked to emotion. Being a proprietary algorithm, there is little information about how these 'frustration' values are determined (further explained in Section 3). Hence, this paper also analyses the raw EEG signals using the standardised low resolution brain electromagnetic tomography (sLORETA) method [16] for spatial analysis. This method was chosen as it yields images of standardised current density with zero localisation error [16] and is an alternative to analysing P300 responses or sub-band frequency powers [17] which are both temporal analysis methods. For an indepth explanation of sLORETA the reader is referred to [16]. The advantage of using spatial analysis is that it can distinguish between regions of temporal activity. The method (sLORETA) helps quantify these active regions of the brain to assist in deducing emotions with the added benefit of visualising brain activity.

\subsection{Eye gaze tracking}

In addition to EEG, eye gaze tracking data can be analysed to identify audio/visual content linked to emotional responses (see Section 5.6). Similar to older EEG headsets, eye gaze trackers consisted of mounting superfluous amounts of equipment on the head such as a camera, glasses, reflective plates and camera mounts $[18,19]$. Recently there have been improvements to eye gaze software which has made it possible to track eye gaze from video without calibration [18] and from standard low resolution

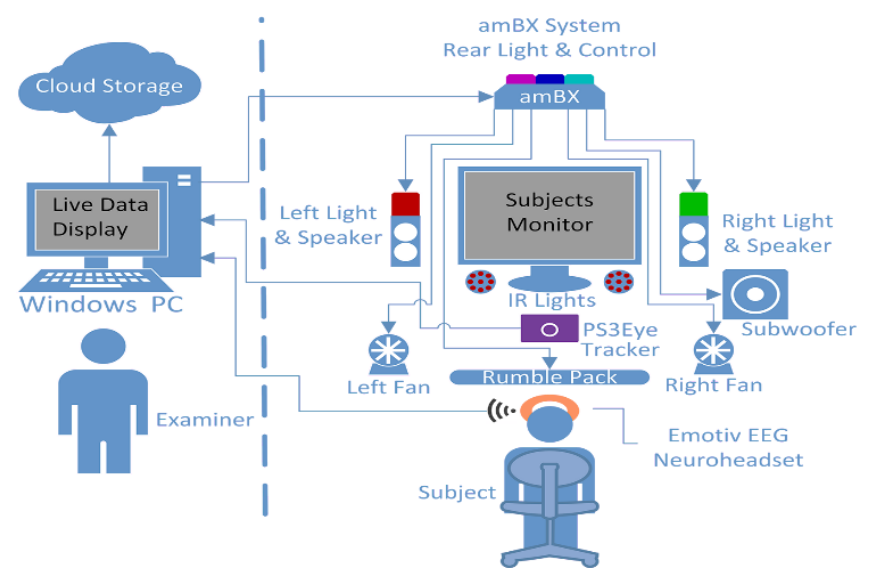

Figure 2: Hardware configuration and test setup

web cameras [20, 21]. Compared to more sophisticated systems involving special glasses or other hardware, later systems minimise eye distractions that may otherwise influence the test results.

To increase the accuracy of the eye gaze tracker, the system used in this paper includes a PS3Eye [22], varifocal lens and an infrared (IR) light source pointing towards the eyes of the subject. The varifocal lens was added to the PS3Eye to improve focus. The PS3Eye manufactured by Sony was chosen as it was designed in collaboration with sensor chip manufacturer OmniVision Technologies to perform well in variable lighting [23]. Hence, this improves the resolution and quality of the image of the eye that is captured due to the accountability of distance to the subject and varying lighting conditions. The high frame rate of this camera (60 frames per second) makes it possible to sample faster and average multiple image frames together to improve the accuracy of the tracker. The IR light source causes only the cornea to reflect the light back at the camera. This creates a distinct pupil in the image which can then be segmented from the image using software algorithms that recognise the pupil and edges of the eye [20]. The work in this paper uses the ITU Gaze Tracker (GT) [24], which has been shown to provide accurate performance [21], to collect eye gaze data as a user views videos.

\section{INTEGRATED BIOSENSOR-BASED QOE EVALUATION SYSTEM}

In order to record all of the information a software package was written which integrates the ITU GT, Emotiv EEG headset and the Philips amBX multisensory media system. The ITU GT was added to the package so that the original interface could be controlled to perform the calibration and recordings. The integrated system is shown in Figure 1.

The Emotiv EEG headset comes with a Software Development Kit (SDK) which allows recording of raw EEG and filtered signals. The software records values from the Expressiv ${ }^{\mathrm{TM}}$ and Affectiv ${ }^{\mathrm{TM}}$ suites, saving information on facial expressions and real-time changes in subjective emotions, respectively. The experiments in this paper analyse the 'frustration' values recorded from the Affectiv ${ }^{\mathrm{TM}}$ suite as well as the raw EEG signals. Emotiv have informally mentioned [25] that Affectiv $^{\mathrm{TM}}$ values are calculated from algorithms written to correlate to subjective studies as explained in [26]. A live display was added to show the values from the Affectiv ${ }^{\mathrm{TM}}$ suite, Expressiv ${ }^{\mathrm{TM}}$ suite, gyroscope and contact quality of the sensors (see Figure 1).

The amBX multisensory media system is packaged with an SDK allowing the software package to control the equipment 


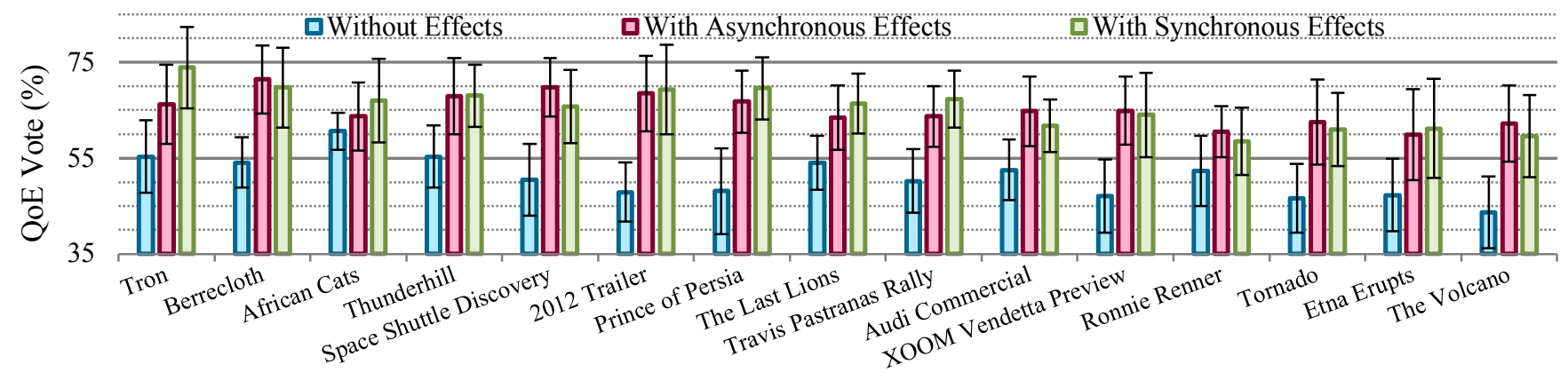

Shortened Video Titles

\section{Figure 3: QoE Votes and 95\% Confidence Interval}

precisely. A media player was coded into the user interface to display audio and video content. The program is coded based on the MPEG-V standard [27] so that it can read vibration, wind and lighting effects from compatible sensory effects metadata files. An auto extraction feature was written for the lighting effects based on previous work [28]. The multisensory media section of the software package records the times when events occur in the video. These times are saved along with the EEG and GT logs relative to a common timestamp.

\section{SUBJECTIVE TESTING METHODOLOGY}

The testing methodology used was designed to coincide with research done in $[2,3,29]$. The design was based on a single stimulus testing method where subjects were introduced to the equipment using a training phase [4]. Videos and their effects were chosen based on $[2,4]$. The asynchronous effects were designed so that all effects (wind, vibration and light) preceded the audio and video by $500 \mathrm{~ms}$. This value was chosen as it is the average skew in the positive and negative direction considered perceptually noticeable for media synchronisation $[11,12]$. This section describes the procedure used to evaluate the subjects with biosensors and relates to previous discrete results [2, 3] by incorporating a QoE voting stage for the videos. The equipment setup and test configuration is depicted in Figure 2.

\subsection{Introduction and pre-questionnaire}

The evaluation begins by describing the procedure, set of tasks and rating method to the subject. The subject is told the working definition of QoE as stated in [30] and that they will be asked to base their votes on their QoE of the presented multisensory media. Following their consent, the evaluation begins. A pre-questionnaire establishes basic demographic data (name, age, gender). This was used to later determine that there were no significant differences in test results due to age or gender [4].

\subsection{Biosensor setup}

The biosensors are set up before the training phase to ensure the participants become familiar with the equipment prior to the main evaluation. The EEG headset is set up first using an appropriate saline solution to ensure sensor contacts are fitted to provide for the highest signal quality. The GT calibration takes place next and the subject is positioned approximately 1 metre from the screen [4]. The camera is optically zoomed so that any black background visible in the image is minimised and the eyes are located near the centre of the image. The colour of the GT calibration background is set to a light grey colour; this allows for miosis in the dim environment which gives a clearer distinction between the sclera, iris and pupil. Individual calibration points are re-calibrated if the GT cannot determine which quadrant of the screen the subject is gazing at.

\subsection{Training phase}

A training phase takes place after the biosensor setup, as recommended in [4]. This is designed to eliminate the surprise effect [4] by helping the participants to become familiar with the stimulus presentation and style of QoE voting. The design was adapted from the training phases used in $[4,29]$. The results of the training phase are not included in the final analysis. The training phase for all evaluations used a shortened version of the publicly available 'Titanic (1997)' trailer (2012 3D release). The genre of the trailer is Drama and is presented with 18 wind effects and 13 vibration effects. The training video was shown consecutively three times with different effects picked randomly from: no effects; asynchronous effects; and synchronous effects. After each video the subject was asked to vote their QoE on an eleven-grade numerical quasi-continuous quality scale from 0 to $100[3,4,31]$.

\subsection{Main evaluation}

Once the subjects are familiar with the equipment, effects and the voting stages, the EEG and GT logging commences for the main evaluation. The 15 videos seen in Figure 3 (dataset available at [32]) are randomly presented three times; without effects, with asynchronous effects and with synchronous effects. No two videos with the same title are shown consecutively [29]. At the end of each video the subject is asked to record their QoE. The voting takes no more than 10 seconds as suggested in [31]. The QoE votes are stored together with logs for the biosensors. The single stimulus assessment method is adopted from [4]. The videos have an average duration of 30 seconds [32] and the entire evaluation takes approximately 22.5 minutes.

\subsection{Post-questionnaire}

At the end of the main evaluation the EEG headset is removed and the subject is asked to complete a post-questionnaire $[2,4]$. The post-questionnaire is given to gain feedback on the evaluation task and gives the subject opportunity to provide recommendations to the procedure.

\section{RESULTS}

For the subjective testing 10 subjects ( 6 males and 4 females) were chosen from an initial set of 15 who were invited to participate in 


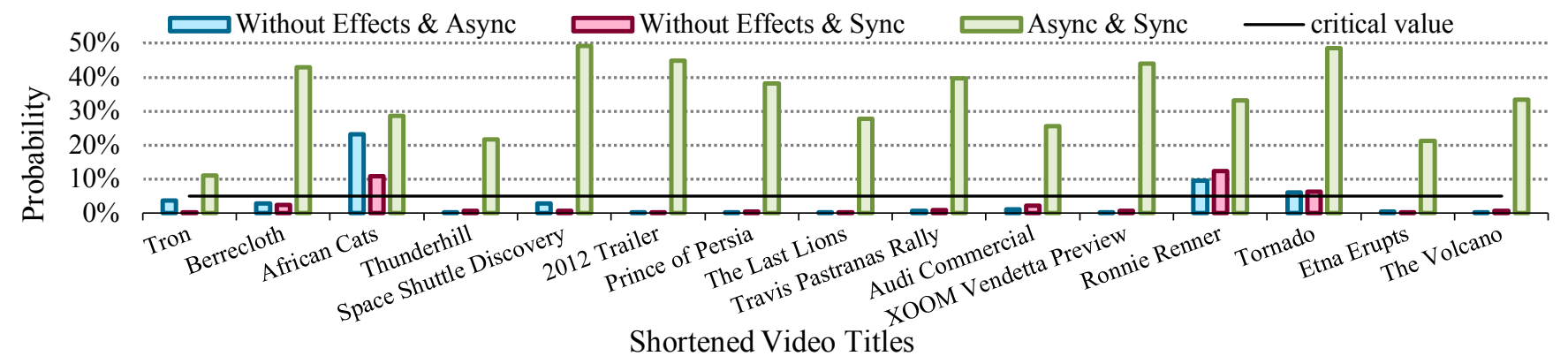

Figure 4: T-Test showing the probability that mean $\mathrm{QoE}$ would be observed the same $($ alpha $=0.05)$

the experiment. Five subjects were excluded based on EEG artefacts. Physiological tests can be highly complex and we found reliability to be a key issue. The mean age for this set of participants is 28.8 and ranges from 19 to 59 with a sample standard deviation $\left(s_{n-1}\right)$ of 12.2. The post-questionnaire showed that some subjects commented on the vibration effect with various recommendations on placement, realism and timing. Some subjects also stated that they thought there was a difference between the videos with synchronous and asynchronous effects; however, they were unsure of exactly what the difference was.

\subsection{Discrete QoE voting}

Each subject voted on their QoE for each video as described in Sections 4.1 and 4.4. The votes were analysed for each video and the Mean Opinion Score (MOS) was plotted for each video and effect type as recommended in [4]. The results for this evaluation can be seen in Figure 3 and are presented with a 95\% confidence interval. Full video titles can be found at [32]. The videos are ordered left to right from highest mean vote to lowest mean vote, respectively.

A single factor Analysis of Variance (ANOVA) was applied to the votes for each video to determine if there was a discernable difference between the different effect types. ANOVA was applied with alpha equal to 0.05 . The p-values of the ANOVA test showed that for $80 \%$ of the videos there was a discernable difference between effect types and so Student's t-tests were then applied to refine the differences.

Figure 4 shows the probabilities calculated using a Student's t-test analysis of the QoE votes [2, 4], using a one tail distribution and a two-sample equal variance. This was conducted three times under the following null hypotheses:

$H_{0_{1}}: \mu_{W o E}=\mu_{\text {Async }} ; H_{0_{2}}: \mu_{W o E}=\mu_{\text {Sync }} ; H_{0_{3}}: \mu_{\text {Async }}=\mu_{\text {Sync }}$ (1)

The mean of the QoE votes is denoted by $\mu_{W o E}, \mu_{A s y n c}$ and $\mu_{\text {Sync }}$ for without effects, with asynchronous effects and with synchronous effects, respectively. The alternative hypotheses for these tests are:

$H_{1_{1}}: \mu_{W o E}<\mu_{\text {Async }} ; H_{1_{2}}: \mu_{W o E}<\mu_{\text {Sync }} ; H_{1_{3}}: \mu_{\text {Async }}<\mu_{\text {Sync }}$

The critical value is $0.05(5 \%)$ and Figure 4 shows that the null hypotheses is rejected $80 \%$ of the time for $H_{0_{1}}$ and $H_{0_{2}}$, and also shows that the alternative hypothesis $H_{1_{3}}$ is rejected $100 \%$ of the time. This shows that videos with effects have a significantly larger mean QoE observed than videos without and agrees with [2$4,28]$. It also shows that there is no significant difference in mean QoE observed between asynchronous and synchronous effects. This indiscernible difference may be due to the asynchronicity being on one side of the perceptual threshold for some effects and on the other for other effects. It may also be possible that the large contrast between no effects and effects reduced the apparent difference between async and sync effects.

\subsection{Temporal physiological responses}

The biosensor analysis was completed with the two videos that had the highest mean vote for QoE. The videos were 'Tron' and 'Berrecloth' with the highest and second highest mean vote, respectively. EEG analysis using 'frustration' values was completed using both videos, whereas, the eye gaze and raw EEG analysis was performed on the 'Tron' video. The data was analysed to show responses directly after major effects. Vibration was found to be the most dominant effect when compared to light and wind in [4] and so the first vibration with $100 \%$ strength was examined.

\subsection{Electroencephalography (EEG)}

For this analysis all 10 sets of data were used to find the 'frustration' values throughout the 'Tron' and 'Berrecloth' videos. Frustration was chosen as it is synonymous to annoyance stated in the working definition of QoE [30]. The description of synchronous effects presented to the subjects for the video 'Tron' and 'Berrecloth' are available at [32]. The EEG data for all subjects was linearly interpolated to a common sampling rate of $10 \mathrm{~Hz}$ and then filtered using a moving average filter with a window size of 10 samples. The filtered data was normalised so that all subjects were within the same range of amplitude and the gradients of this information was calculated. The frustration gradients can be related to the time that the effects occurred using the effect metadata.

To calculate whether there is a significant difference with increasing and decreasing frustration a statistical analysis needs to be applied. The same method is adopted from the one described in Section 5.1, however, it is now two-tailed and is applied to the frustration gradients between the start of the first full strength vibration and second full strength vibration. The null hypotheses for this test asked if the frustration gradients are observed statistically greater than zero. The results of the t-tests show that $30 \%$ of subjects were observed having a significant increase in frustration for no effects and asynchronous effects and 50\% for synchronous.

The analysis used for 'Tron' was then applied to 'Berrecloth'. The period is once again equal to the gap between the first and second full strength vibrations. The results of the t-tests show that $10 \%$ of subjects were observed having a significant increase in frustration for no effects and synchronous effects and $30 \%$ for asynchronous. Frustration in these cases may be caused by unanticipated and/or unfavourable effects. It is difficult to draw conclusions due to the 'frustration' algorithm being proprietary and so this paper explores the recorded EEG potentials. 


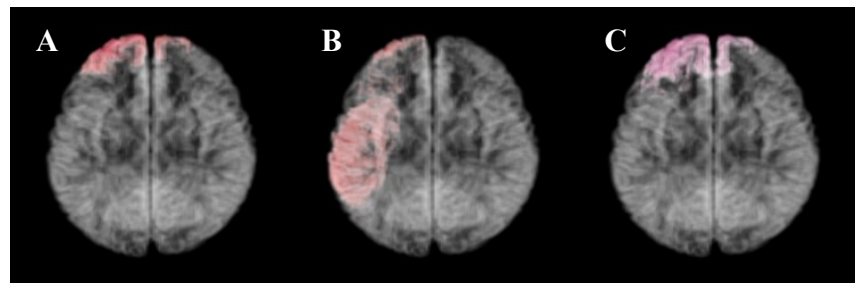

Figure 5: Subject with increased brain activity in the temporal lobe. No effects (A), async effects (B) and sync effects (C)

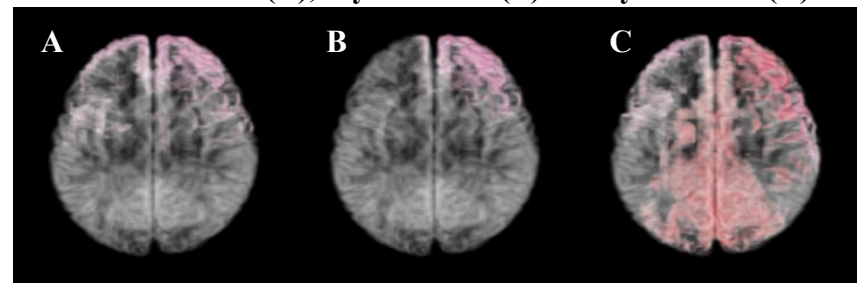

Figure 6: Subject with increased brain activity in the occipital lobe. No effects (A), async effects (B) and sync effects (C)

\subsection{Spatial EEG analysis}

The sLORETA method was used to analyse the raw EEG data due to the proprietary Affectiv ${ }^{\mathrm{TM}}$ algorithms. This method was used to determine the location at which the propagating electrical potentials originated. Data from the 'Tron' video was used for this analysis due to the stronger first vibration and higher QoE vote. The sLORETA method can provide images of standardised current density and quantification of brain lobes and Brodmann areas. The data was analysed using a moving window with a period of $0.5 \mathrm{~s}$ which overlapped for the duration of the effect and after the effect. Averaging was applied to the EEG signal for 10 s to remove DC bias and a Fast Fourier Transform (FFT) was applied for Delta, Theta, Alpha and Beta bands. The images presented in Figure 5 and Figure 6 show a model brain at 5\% opacity from directly above with the frontal lobe positioned at the top. The coloured areas show the most active regions of the brain with a threshold of $25 \%$. These images are provided in two dimensions (2D) for print; however, three dimensional (3D) visualisations are possible.

\subsection{Synchronicity}

The temporal lobe of the brain can be related to memory of temporal events and the senses these stimulate, which can be related to the synchronicity of the sensory effects. Figure 5 shows a particular subjects brain for the three different versions of the video 'Tron', where it can be seen that for asynchronous effects the temporal lobe is the most active. Furthermore, Figure 7 shows there is an increase of $25 \%$ of activity in the temporal lobe for asynchronous effects for all subjects, whereas, there was no activity in this lobe observed under the conditions for no effects and synchronous effects. There is also an increase in activity in the order of $20-25 \%$ for the occipital lobe during synchronous effects; this is discussed in more detail in 5.6.

\subsection{Eye gaze tracking}

A method with preliminary results for correlating brain lobe activity with gaze deviation is presented in this section. Eye gaze analysis was performed on two individual subjects, one female and one male, because during the videos it was common for subjects to drift off camera causing distorted eye gaze logs. This could be circumvented by keeping subjects still for the length of the test,

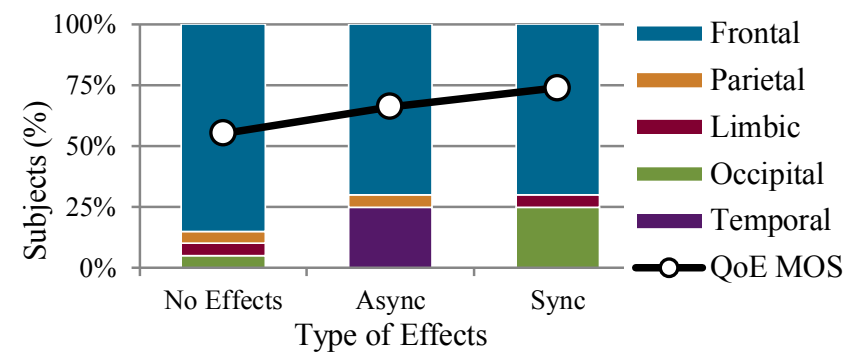

Figure 7: Most active brain lobes for different effects

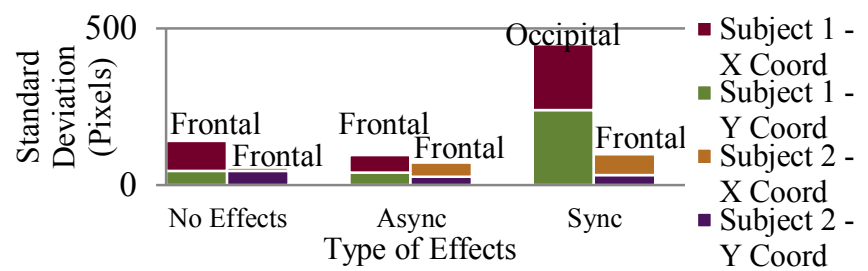

Figure 8: Standard deviations of gaze location with most active brain lobe labelled

reducing the length of the test and/or eye tracking with a wider view. The two individuals chosen had the least distorted eye gaze.

Blinking caused null values which were removed using linear interpolation. The data was then up sampled and filtered using the same methods for the EEG data in Section 5.3. It should be noted that these two subjects had different opinions of the sensory effects; this was apparent from the subject's QoE votes. Named subject one and two, they are situated on the left and right side for each effect type in Figure 8, respectively. Subject one had an average QoE vote of 47,69 and 68, and subject two, 54, 44 and 45 for no effects, async effects and sync effects, respectively. The order of the videos was async effects, no effects and sync effects for subject one and async effects, sync effects and no effects for subject two.

A much larger standard deviation for synchronous effects is experienced by subject one but only slightly for subject two. At this time and for this effect, subject one also has more activity in the occipital lobe (Figure 6) which may be correlated to the increased gaze deviation. A significant aspect of the occipital lobe is the primary visual cortex, which highly correlates to increased gaze deviation. Subject two lacks activity in this lobe and this is reflected in the uniform gaze deviation across effect types.

\section{CONCLUSIONS}

This paper presents results for QoE assessment of multisensory media using an EEG neuroheadset and eye gaze tracker. The response to sensory effects when complementing audio/visual content under asynchronous and synchronous conditions is compared to content with no effects. The results show that sensory effects enhance the QoE; however, there was statistically indiscernible difference between the synchronicities of effects. Furthermore, the EEG results show that there is correlating brain activity with a $20-25 \%$ decrease in frontal lobe activity for both asynchronous and synchronous effects. The EEG results also show that there is an increase in activity in the temporal lobe by $25 \%$ for asynchronous and occipital lobe by $20 \%$ for synchronous. The preliminary gaze tracker results may support this by showing that gaze deviation and occipital lobe activity increase mutually.

Future work includes identifying the influence of a wider range of synchronicities on QoE by conducting further user studies and data mining to gather information of alternate correlations 
between biosensors and QoE thus providing better knowledge for future user studies in the area.

\section{ACKNOWLEDGEMENTS}

The authors would like to thank all participants for volunteering their time, the Commonwealth Scientific and Industrial Research Organisation (CSIRO) for their help with EEG analysis and Christian Timmerer and Benjamin Rainer for their insightful and useful preliminary discussions related to this work.

\section{REFERENCES}

[1] C. Timmerer, M. Waltl, B. Rainer, and N. Murray, "Sensory Experience: Quality of Experience Beyond Audio-Visual," in Quality of Experience, ed: Springer, 2014, pp. 351-365.

[2] B. Rainer, M. Waltl, E. Cheng, M. Shujau, C. Timmerer, S. Davis, et al., "Investigating the impact of sensory effects on the quality of experience and emotional response in web videos," in Quality of Multimedia Experience (QoMEX), 2012 Fourth International Workshop on, 2012, pp. 278-283.

[3] M. Waltl, C. Timmerer, and H. Hellwagner, "Improving the quality of multimedia experience through sensory effects," in Quality of Multimedia Experience (QoMEX), 2010 Second International Workshop on, 2010, pp. 124-129.

[4] C. Timmerer, B. Rainer, and M. Waltl, "A utility model for sensory experience," in Quality of Multimedia Experience (QoMEX), 2013 Fifth International Workshop on, 2013, pp. 224-229.

[5] C. Timmerer, M. Waltl, B. Rainer, and H. Hellwagner, "Assessing the quality of sensory experience for multimedia presentations," Signal Processing: Image Communication, vol. 27, pp. 909-916, 2012.

[6] P. C. Petrantonakis and L. J. Hadjileontiadis, "Emotion recognition from brain signals using hybrid adaptive filtering and higher order crossings analysis," Affective Computing, IEEE Transactions on, vol. 1, pp. 81-97, 2010.

[7] R. B. Silberstein and G. E. Nield, "Measuring Emotion in Advertising Research: Prefrontal Brain Activity," Pulse, IEEE, vol. 3, pp. 24-27, 2012.

[8] J.-N. Antons, S. Arndt, R. Schleicher, and S. Möller, "Brain Activity Correlates of Quality of Experience," in Quality of Experience, ed: Springer, 2014, pp. 109-119.

[9] E. Cheng, S. Davis, I. Burnett, and C. Ritz, "An ambient multimedia user experience feedback framework based on user tagging and EEG biosignals," 4th International Workshop on Semantic Ambient Media Experience (SAME 2011), pp. 1-5, 2011.

[10] J. G. Carrier, "Mind, Gaze and Engagement Understanding the Environment," Journal of Material Culture, vol. 8, pp. 523, 2003.

[11] R. Steinmetz, "Human perception of jitter and media synchronization," Selected Areas in Communications, IEEE Journal on, vol. 14, pp. 61-72, 1996.

[12] W. Yaodu, X. Xiang, K. Jingming, and H. Xinlu, "A speechvideo synchrony quality metric using CoIA," in Packet Video Workshop (PV), 2010 18th International, 2010, pp. 173-177.

[13] V. Jurcak, D. Tsuzuki, and I. Dan, "10/20, 10/10, and 10/5 systems revisited: their validity as relative head-surface-based positioning systems," Neuroimage, vol. 34, pp. 1600-1611, 2007.

[14] Emotiv. (2014, May). EEG Features [Online]. Available: http://emotiv.com/eeg/
[15] M. S. Buchsbaum, "Frontal cortex function," American Journal of Psychiatry, vol. 161, pp. 2178-2178, 2004.

[16] R. Pascual-Marqui, "Standardized low-resolution brain electromagnetic tomography (sLORETA): technical details," Methods Find Exp Clin Pharmacol, vol. 24, pp. 5-12, 2002.

[17] R. Gupta, S. Arndt, J.-N. Antons, R. Schleicher, S. Moller, and T. H. Falk, "Neurophysiological experimental facility for Quality of Experience (QoE) assessment," in Integrated Network Management (IM 2013), 2013 IFIP/IEEE International Symposium on, 2013, pp. 1300-1305.

[18] S. Kohlbecher, S. Bardinst, K. Bartl, E. Schneider, T. Poitschke, and M. Ablassmeier, "Calibration-free eye tracking by reconstruction of the pupil ellipse in 3D space," in Proceedings of the 2008 symposium on Eye tracking research \& applications, 2008, pp. 135-138.

[19] C. W. Huang, Z. S. Jiang, W. F. Kao, and Y. L. Huang, "Building a Low-Cost Eye-Tracking System," Applied Mechanics and Materials, vol. 263, pp. 2399-2402, 2013.

[20] R. Valenti, J. Staiano, N. Sebe, and T. Gevers, "Webcambased visual gaze estimation," Image Analysis and Processing-ICIAP 2009, pp. 662-671, 2009.

[21] J. San Agustin, H. Skovsgaard, E. Mollenbach, M. Barret, M. Tall, D. W. Hansen, et al., "Evaluation of a low-cost opensource gaze tracker," in Proceedings of the 2010 Symposium on Eye-Tracking Research \& Applications, 2010, pp. 77-80.

[22] Sony. (2014, April 24). PS3 EYE CAMERA [Online]. Available: http://www.sony.com.au/product/playstation+eye

[23] N. G. Croal. (2007, May). Geek Out: The Playstation Eye is Nearly Upon Us. Dr. Richard Marks Takes Us Behind the Scenes of its Birth. [Online]. Available: http://archive.today/WESxm

[24] ITUGazeGroup. (2014, May). ITU Gaze Tracker [Online]. Available: http://www.gazegroup.org/downloads/23

[25] Emotiv. (2014, July). Scientific background for the emotions in the affectiv suite, what is it? [online]. Available: emotiv.com/forum/messages/forum4/topic1262/message7424/

[26] K. M. Gilleade and A. Dix, "Using frustration in the design of adaptive videogames," in Proceedings of the 2004 ACM SIGCHI International Conference on Advances in computer entertainment technology, 2004, pp. 228-232.

[27] C. Timmerer, S. Hasegawa, and S. Kim, "Working Draft of ISO/IEC 23005 Sensory Information," ISO/IEC JTC 1/SC 29/WG 11 N, vol. 10618, 2009.

[28] M. Waltl, C. Timmerer, and H. Hellwagner, "Increasing the user experience of multimedia presentations with sensory effects," in Image Analysis for Multimedia Interactive Services (WIAMIS), 2010 11th International Workshop on, 2010, pp. 1-4.

[29] F. De Simone, M. Naccari, M. Tagliasacchi, F. Dufaux, S. Tubaro, and T. Ebrahimi, "Subjective assessment of $\mathrm{H}$. 264/AVC video sequences transmitted over a noisy channel," in Quality of Multimedia Experience, 2009. QoMEx 2009. International Workshop on, 2009, pp. 204-209.

[30] P. Le Callet, S. Möller, and A. Perkis, "'Qualinet White Paper on Definitions of Quality of Experience," European Network on Quality of Experience in Multimedia Systems and Services (COST Action IC 1003), Lausanne, Switzerland, Tech. Rep, vol. 1.2, p. 6, 2013.

[31] P. ITU-T RECOMMENDATION, "Subjective video quality assessment methods for multimedia applications," 1999.

[32] (2014, April). Sensory Experience Lab (SELab). Available: http://selab.itec.aau.at 